\title{
Some Findings on Genes over SARS-CoV2 Genomes
}

\author{
Sk. Sarif Hassan ${ }^{\mathrm{a}, *}$, Atanu Moitra ${ }^{\mathrm{b}}$, Pabitra Pal Choudhury ${ }^{\mathrm{c}}$, Prasanta \\ Pramanik $^{\mathrm{d}}$, Siddhartha Jana ${ }^{\mathrm{e}}$ \\ ${ }^{a}$ Department of Mathematics, Pingla Thana Mahavidyalaya, Maligram 721140, India \\ ${ }^{b}$ CMO, Government of West Bengal, India. \\ ${ }^{c}$ Applied Statistics Unit, Indian Statistical Institute, Kolkata 700108, West Bengal, India. \\ ${ }^{d}$ Finance Department, Government of West Bengal, India. \\ ${ }^{e}$ School of Biological Sciences, Indian Association for the Cultivation of Science, West \\ Bengal, 700032, India.
}

\begin{abstract}
Coronaviruses are a large family of RNA viruses which cause respiratory infections ranging from the common cold to more severe diseases such as Middle East Respiratory Syndrome (MERS), Severe Acute Respiratory Syndrome (SARS) and COVID-19. This article highlights some key findings based on a thorough scanning of genes of 470 SARS-CoV2 genomes, including the co-presence of ORF7a and ORF8 over the 251 SARS-CoV2 genomes and the absence of the gene ORF7b over the 219 SARS-CoV2 genomes collected from various countries including India.
\end{abstract}

Keywords: SARS-CoV2; ORF7a; ORF7b; ORF8; SARS-CoV2 Genomes.

\section{Introduction}

The outbreak of the SARS-CoV2, a novel coronavirus becomes now a pandemic 1, 2. The genome of SARS-CoV2 contains approximately 30kbp nucleotides and each genomes contains around 11 genes of various type such as $\mathrm{S}$,

\footnotetext{
${ }^{*}$ Corresponding author

Email addresses: sarimif@gmail.com (Sk. Sarif Hassan ), atanu.mli@gmail.com (Atanu Moitra), pabitrapalchoudhury@gmail.com (Pabitra Pal Choudhury),

prasanta.pramanik@gmail.com (Prasanta Pramanik), siddhartha.jana@gmail.com (Siddhartha Jana)
} 
5 E, M, N etc [3]. The SARS-CoV2 genomes consist of structural protein gene S which specifically bind to the receptor of the host cell, and this is the key protein for viruses to invade susceptible cells. The gene $\mathrm{M}$ and $\mathrm{E}$ are involved in the formation of the virus envelope, while the gene $\mathrm{N}$ is involved in the assembly of the virus [4. The origin of the source of the virus SARS-CoV2 and its intermediate host is still controversial [5]. The phylogenetic analysis implied that the coronavirus was the most similar to Bat coronavirus isolate RaTG13 (GenBank No.: MN996532), with $96.2 \%$ nucleotide homology in the whole genome [5]. It is also reported that the SARS-CoV2 was closely related (with $88 \%$ identity) to two bat-derived severe acute respiratory syndrome (SARS)-like coronaviruses, bat-SL-CoVZC45 and bat-SL-CoVZXC21, collected in 2018 in Zhoushan, eastern China, but were more distant from SARS-CoV (about 79\%) and MERS$\mathrm{CoV}$ (about 50\%) 6]. It is reported that the genome sequences MT050493, MT012098 from India are highly similar to the genome of the Wuhan seafood market pneumonia virus (accession number: NC 045512) [7]. The other recent findings and present state of the art including review can be obtained from the various articles [8, 9, 10, 11, 12, 13, 14, 15, 16, 17, 18.

Replication of Coronavirus is caused by a set of highly conserved viral proteins. Only two ORFs such as ORF3a and ORF7a have been in virus-infected cells among the eight putative accessory proteins encoded by the (SARS-CoV)

25 [19. The ORF7b gene is expressed in virus-infected cell lysates and from a cDNA encoding the gene 7 coding region, indicating that the sgRNA7 is bicistronic. The ORF7b protein is not only an accessory protein but a structural component of the SARS-CoV virion [19].

It is needless to mention that a deep scanning over these genomes and as-

30 sociated genes is necessary for various reasons including pathogenesis [3]. In this article, an attempt has been made to search out the gene variations among 
the 470 SARS-CoV2 genomes. This article reports that a pair of genes viz. ORF7a and ORF8 is present across almost all the SARS-CoV2 genomes except MN988668, MN988669 and MT121215 genomes from China. Also it is found 35 that ORF7b is absent across 219 genomes of SARS-CoV2 such as MT050493 (India), MT012098 (India).

\subsection{Dataset}

As on date 14th April, 2020, 470 complete genome sequences of SARS-CoV2 were available in the NCBI virus database. From the NCBI Virus Database

(https : //www.ncbi.nlm.nih.gov/labs/virus/vssi/) we fetch all the 470 genomes and their associated genes from each genomes. The accession of the 470 genomes are given in the Table-1. The known gene-organization of a typical SARS-CoV2 genomes is given in the Fig. 1. The first ORF representing approximately $67 \%$ of the entire genome encodes 16 non-structural proteins (nsps), while the 45 remaining ORFs encode accessory proteins and structural proteins [20].

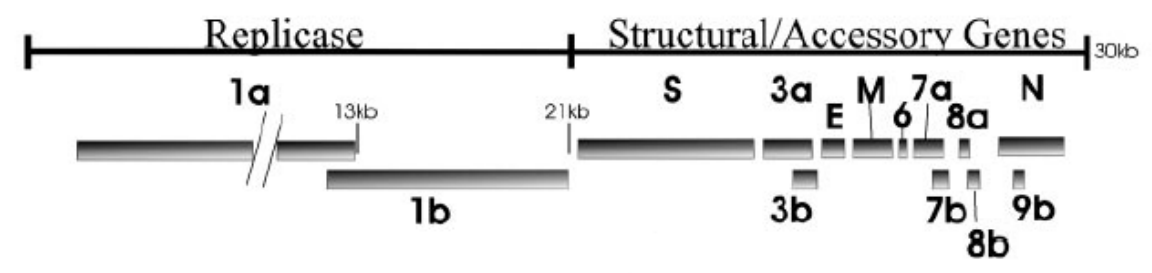

Figure 1: Gene-organizations over a typical SARS-CoV2 genome. [19] 
Table 1: List of the 470 SARS-CoV2 Genome Accessions (Data collected on 14th April, 2020)

\begin{tabular}{|c|c|c|c|c|c|}
\hline Accession & Accession & Accession & Accession & Accession & Accession \\
\hline 045512 & MT291836 & MT262912 & MT263440 & MT253707 & MT163716 \\
\hline MT077125 & MT291831 & MT262913 & MT263447 & MT251972 & MT159706 \\
\hline MT039874 & MT293170 & MT262914 & MT263449 & MT251974 & MT159716 \\
\hline MT322402 & MT292574 & MT262993 & MT263455 & MT251975 & MT159719 \\
\hline MT322407 & MT293178 & MT263074 & MT263444 & MT251973 & MT159707 \\
\hline MT322396 & MT293181 & MT263381 & MT263445 & MT251976 & MT159717 \\
\hline MT322394 & MT293183 & MT263391 & MT263451 & MT251979 & MT159709 \\
\hline MT322397 & MT293195 & MT262901 & MT263420 & MT253697 & MT159715 \\
\hline MT322398 & MT293196 & MT262903 & MT263441 & MT253699 & MT159718 \\
\hline MT322400 & MT293197 & MT262907 & MT263454 & MT253696 & MT159722 \\
\hline MT322401 & MT293204 & MT262909 & MT263464 & MT253698 & MT159708 \\
\hline MT322403 & MT293212 & MT262911 & MT263465 & MT253700 & MT159705 \\
\hline MT322404 & MT293215 & MT262899 & MT263468 & MT251977 & MT159710 \\
\hline MT322405 & MT293216 & MT262904 & MT263394 & MT251978 & MT159711 \\
\hline MT322406 & MT293219 & MT262915 & MT263395 & MT251980 & MT159712 \\
\hline MT322408 & MT293224 & MT262916 & MT263396 & MT233526 & MT159713 \\
\hline MT322409 & MT293225 & MT262897 & MT259226 & MT246667 & MT159714 \\
\hline MT322410 & MT293206 & MT262898 & MT259275 & MT246451 & MT159720 \\
\hline MT322411 & MT293208 & MT262905 & MT259247 & MT246461 & MT159721 \\
\hline MT322412 & MT293209 & MT262910 & MT258377 & MT246471 & MT121215 \\
\hline MT322413 & MT295464 & MT263400 & MT258378 & MT246472 & MT066156 \\
\hline MT322414 & MT293160 & MT263382 & MT258379 & MT246474 & MT050493 \\
\hline MT322415 & MT293166 & MT263383 & MT259231 & MT246450 & MT012098 \\
\hline MT322416 & MT293171 & MT263384 & MT259228 & MT246453 & MT152824 \\
\hline MT322417 & MT293190 & MT262896 & MT259248 & MT246454 & MT135044 \\
\hline MT322418 & MT293161 & MT263415 & MT259227 & MT246462 & MT135042 \\
\hline MT322419 & MT293167 & MT263406 & MT259236 & MT246464 & MT135041 \\
\hline MT322420 & MT293168 & MT263408 & MT258380 & MT246470 & MT135043 \\
\hline MT322421 & MT293174 & MT263422 & MT259235 & MT246473 & MT126808 \\
\hline MT322422 & MT293175 & MT263469 & MT259237 & MT246480 & MT123293 \\
\hline MT322423 & MT293182 & MT263439 & MT259239 & MT246484 & MT123291 \\
\hline MT322424 & MT293191 & MT263457 & MT259281 & MT246449 & MT123290 \\
\hline MT322395 & MT293158 & MT263459 & MT259282 & MT246455 & MT123292 \\
\hline MT320538 & MT293162 & MT263432 & MT259243 & MT246456 & MT118835 \\
\hline MT320891 & MT293163 & MT263450 & MT259249 & MT246478 & MT106052 \\
\hline MT308702 & MT293164 & MT263458 & MT259250 & MT246485 & MT106053 \\
\hline MT308703 & MT281577 & MT263467 & MT259251 & MT246488 & MT106054 \\
\hline MT308704 & MT293156 & MT263411 & MT259256 & MT246452 & MT093571 \\
\hline MT304476 & MT293159 & MT263413 & MT259258 & MT246460 & MT093631 \\
\hline MT304474 & MT291834 & MT263426 & MT259266 & MT246481 & MT072688 \\
\hline MT304475 & MT291829 & 3421 & IT259267 & $\mathrm{M}$ & MT066176 \\
\hline MT304478 & MT291827 & MT263443 & MT259274 & MT246490 & MT066175 \\
\hline MT304482 & MT291830 & MT263412 & MT259286 & MT246459 & MT044258 \\
\hline MT304488 & MT291828 & MT263416 & MT259287 & MT246468 & MT044257 \\
\hline MT304479 & MT293169 & MT263417 & MT259241 & MT246475 & MT049951 \\
\hline MT304480 & MT293200 & MT263423 & MT258381 & MT246477 & MT039887 \\
\hline MT304481 & MT293210 & MT263431 & MT259257 & MT246479 & MT039888 \\
\hline MT304486 & MT293211 & MT263410 & MT259261 & MT246457 & MT039890 \\
\hline MT304487 & MT293218 & MT263424 & MT259263 & MT246458 & MT039873 \\
\hline MT304490 & MT295465 & MT263425 & MT259264 & MT246466 & MT027062 \\
\hline MT304491 & MT293198 & MT263442 & MT259268 & MT246467 & MT027063 \\
\hline MT304483 & MT293205 & MT263402 & MT259269 & MT246469 & MT027064 \\
\hline MT304484 & MT293207 & MT263405 & MT259271 & MT246476 & MT020881 \\
\hline MT304485 & MT293213 & MT263418 & MT259273 & MT246486 & MT019530 \\
\hline MT304489 & MT293220 & MT263419 & MT259277 & MT246487 & MT019531 \\
\hline MT300186 & MT293222 & MT263398 & MT259278 & MT246489 & MT019533 \\
\hline MT304477 & MT292569 & MT263399 & MT259280 & MT240 & MT020880 \\
\hline MT292571 & MT293172 & MT263403 & MT258383 & MT233523 & MT019532 \\
\hline MT293186 & MT293177 & MT263404 & MT258382 & MT233519 & MT019529 \\
\hline MT292570 & MT293176 & MT263414 & MT259246 & MT23 & MT007544 \\
\hline MT292573 & MT293199 & MT263430 & MT259244 & MT226610 & MN996531 \\
\hline MT293173 & MT293165 & MT263390 & MT259245 & MT198652 & MN996530 \\
\hline MT292575 & MT276597 & MT263434 & MT259252 & MT192773 & MN996527 \\
\hline MT293179 & MT276598 & MT263436 & MT259253 & MT192772 & MN996528 \\
\hline MT293180 & MT276323 & MT263446 & MT259254 & MT192765 & MN996529 \\
\hline MT293184 & MT276328 & 2634 & MT259284 & MT192759 & MN997409 \\
\hline MT293189 & MT276331 & MT263452 & MT259229 & MT188341 & MN988668 \\
\hline MT293192 & MT276329 & MT263453 & MT259230 & MT188340 & MN988669 \\
\hline MT293194 & MT276330 & MT263456 & MT259260 & MT188339 & MN994467 \\
\hline MT293201 & MT276324 & MT263462 & MT259285 & MT184909 & MN994468 \\
\hline MT293202 & MT276325 & MT263463 & MT253710 & MT184911 & MN988713 \\
\hline MT292572 & MT276327 & MT263386 & MT253709 & MT184912 & MN938384 \\
\hline MT293185 & MT276326 & MT263387 & MT253705 & MT184913 & MN975262 \\
\hline MT293187 & MT263388 & MT263428 & MT253708 & MT184910 & MN985325 \\
\hline MT293188 & MT263392 & IT263420 & MT253701 & MT184907 & MN908947 \\
\hline 291826 & 2900 & $M$ & 253702 & MT184908 & \\
\hline MT291832 & MT262902 & MT263435 & MT253703 & MT163718 & \\
\hline MT291833 & MT262906 & MT263437 & MT253704 & MT163719 & \\
\hline MT291835 & MT262908 & MT263438 & MT253706 & MT163717 & \\
\hline
\end{tabular}




\section{Findings}

Based on a thorough scanning over the set of genes of all the 470 genomes, the following observations are made:

- A pair of genes ORF7a and ORF8 of length 366 is present among almost all the SARS-CoV2 genomes except three genomes viz. MN988668 (China), MN988669 (China) and MT121215 (China). The loci of these two genes across the all the 467 genomes is gapped by $135 \mathrm{bp}$.

- There are 251 genomes out of the 470 genomes that contain a gene ORF7b of length $132 \mathrm{bp}$, located in between the gap-frame of 135bp as mentioned above. The presence of ORF7b over 251 genomes across different geolocations is given in Table 2. It is worth observing from the reference [19] that the presence of ORF7b gene makes a $\mathrm{CoV}$ protein more virulent.

- There are 219 SARS-CoV2 genomes out of 470, which do not contain the ORF7b gene. The list of the genomes with their respective geographic locations, which do not contain ORF7b is given in the Table-3. The absence of ORF7b over 251 genomes across different countries is given in Table 2. It is to be noted that $55.25 \%$ of the SARS-CoV2 genomes from USA contain ORF7b gene which may be an indication of the strong pandemic situation in USA.

- The gene E of length 228 is present over all the 470 SARS-CoV2 genomes.

- The gene M of length 669 is present over all the 470 SARS-CoV2 genomes.

- The gene N of length 1260 is present over all the 470 SARS-CoV2 genomes.

- The spike gene $\mathrm{S}$ is a necessary structural gene and is present over all 470 genomes consisting of gene length $3822 \mathrm{bp}$ for 468 genomes and 3819bp for 
only two genomes, one from India MT012098 and other one from France MT320538.

- There are only three genomes MN988668 (China), MN988669 (China), MT121215 (China) have only five genes (ORFs) viz. ORF1ab, S, E, M and $\mathrm{N}$.

- There are 245 genomes having exactly 11 genes viz. ORF1ab, S, ORF3a, E, M, ORF6, ORF7a, ORF7b, ORF8, N and ORF10.

- There are 221 genomes having exactly 10 genes viz. ORF1ab, S, ORF3a, E, M, ORF6, ORF7a, ORF8, N and ORF10. It is noted that ORF7b is absent in this list of ten genes.

- There is only one genome MN938384 (China) consisting exactly nine genes viz. ORF1ab, S, ORF3, E, M, ORF6, ORF7, ORF8 and N.

Table 2: Presence and Absence of ORF7b over 470 SARS-CoV2 Genomes

\begin{tabular}{ccc}
\hline Country & Absent of ORF7b & Present of ORF7b \\
\hline USA & 166 & 205 \\
China & 36 & 28 \\
Italy & 1 & 1 \\
France & 0 & 1 \\
Iran & 0 & 1 \\
South Korea & 1 & 3 \\
Spain & 4 & 7 \\
Israel & 0 & 2 \\
Pakistan & 0 & 2 \\
Peru & 0 & 1 \\
Viet Nam & 2 & 0 \\
Taiwan & 3 & 0 \\
India & 2 & 0 \\
Brazil & 1 & 0 \\
Sweden & 1 & 0 \\
Nepal & 1 & 0 \\
Australia & 1 & 0 \\
\hline
\end{tabular}

From the Table-2, it is inferred that screening of ORF7b specific test may provide the rate of SARS-CoV2's infectivity and mortality in a country. 
Table 3: List of the genome accessions with their respective geographic location, which do not contain the gene ORF7b

\begin{tabular}{|c|c|c|c|c|c|}
\hline Accession & Geo Location & Accession & Geo Location & Accession & Geo Location \\
\hline MT262896 & USA & MT251976 & USA & MT159709 & USA \\
\hline MT262900 & USA & MT251977 & USA & MT159710 & USA \\
\hline MT262901 & USA & MT251978 & USA & MT159711 & USA \\
\hline MT262902 & USA & MT251979 & USA & MT159712 & USA \\
\hline MT262903 & USA & MT251980 & USA & MT159713 & USA \\
\hline MT262906 & USA & MT253696 & China & MT159714 & USA \\
\hline MT262907 & USA & MT253697 & China & MT159705 & USA \\
\hline MT262908 & USA & MT253698 & China & MT159706 & USA \\
\hline MT262909 & USA & MT253699 & China & MT159716 & USA \\
\hline MT262911 & USA & MT251972 & USA & MT159717 & USA \\
\hline MT262912 & USA & MT251973 & USA & MT159719 & USA \\
\hline MT262913 & USA & MT251974 & USA & MT159707 & USA \\
\hline MT262914 & USA & MT251975 & USA & MT121215 & China \\
\hline MT262897 & USA & MT253700 & China & MT066156 & Italy \\
\hline MT262898 & USA & MT198652 & Spain & MT012098 & India \\
\hline MT262899 & USA & MT233526 & USA & MT050493 & India \\
\hline MT262904 & USA & MT246667 & USA & MT152824 & USA \\
\hline MT262905 & USA & MT246450 & USA & MT135042 & China \\
\hline MT262910 & USA & MT246452 & USA & MT135041 & China \\
\hline MT262915 & USA & MT246453 & USA & MT135043 & China \\
\hline MT262916 & USA & MT246454 & USA & MT135044 & China \\
\hline MT259281 & USA & MT246460 & USA & MT126808 & Brazil \\
\hline MT259282 & USA & MT246461 & USA & MT123293 & China \\
\hline MT258377 & USA & MT246462 & USA & MT123290 & China \\
\hline MT258378 & USA & MT246464 & USA & MT123291 & China \\
\hline MT258379 & USA & MT246470 & USA & MT123292 & China \\
\hline MT259257 & USA & MT246471 & USA & MT118835 & USA \\
\hline MT259261 & USA & MT246449 & USA & MT106052 & USA \\
\hline MT259263 & USA & MT246473 & USA & MT106053 & USA \\
\hline MT259264 & USA & MT246474 & USA & MT106054 & USA \\
\hline MT259268 & USA & MT246455 & USA & MT093571 & Sweden \\
\hline MT259269 & USA & MT246456 & USA & MT072688 & Nepal \\
\hline MT259271 & USA & MT246480 & USA & MT066175 & Taiwan \\
\hline MT259273 & USA & MT246457 & USA & MT066176 & Taiwan \\
\hline MT259275 & USA & MT246458 & USA & MT044258 & USA \\
\hline MT259277 & USA & MT246481 & USA & MT044257 & USA \\
\hline MT259278 & USA & MT246451 & USA & MT039890 & South Korea \\
\hline MT259280 & USA & MT246472 & USA & MT039887 & $\begin{array}{l}\text { USA } \\
\text { USA }\end{array}$ \\
\hline MT258383 & USA & MT246486 & USA & MT039888 & USA \\
\hline MT259247 & USA & MT246487 & USA & MT039873 & China \\
\hline MT259248 & USA & MT246488 & USA & MT027062 & USA \\
\hline MT259236 & USA & MT246489 & USA & MT027063 & USA \\
\hline MT258380 & USA & MT246485 & USA & MT027064 & USA \\
\hline MT259235 & USA & MT246459 & USA & MT019529 & China \\
\hline MT259237 & USA & MT246482 & USA & MT020881 & USA \\
\hline MT259239 & USA & MT246466 & USA & MT019530 & China \\
\hline MT258381 & USA & MT246467 & USA & MT019531 & China \\
\hline MT259243 & USA & MT246484 & USA & MT019533 & China \\
\hline MT259249 & USA & MT246468 & USA & MT020880 & USA \\
\hline MT259250 & USA & MT246490 & USA & MT019532 & China \\
\hline MT259251 & USA & MT246469 & USA & MT007544 & Australia \\
\hline MT259256 & USA & MT246475 & USA & MN988668 & China \\
\hline MT259258 & USA & MT246477 & USA & MN988669 & China \\
\hline MT259266 & USA & MT246478 & USA & MN994467 & USA \\
\hline MT259267 & USA & MT246479 & USA & MN994468 & USA \\
\hline MT259274 & USA & MT246476 & USA & MN997409 & USA \\
\hline MT259286 & USA & MT233519 & Spain & MN988713 & USA \\
\hline MT259287 & USA & MT233522 & Spain & MN985325 & USA \\
\hline MT259241 & USA & MT233523 & Spain & MN975262 & China \\
\hline MT259260 & USA & MT226610 & China & MN938384 & China \\
\hline MT259284 & USA & MT192773 & Viet Nam & MN908947 & China \\
\hline MT259285 & USA & MT192759 & Taiwan & & \\
\hline MT258382 & USA & MT192772 & Viet Nam & & \\
\hline MT259246 & USA & MT188341 & USA & & \\
\hline MT259244 & USA & MT188339 & USA & & \\
\hline MT259245 & USA & MT188340 & USA & & \\
\hline MT259252 & USA & MT184909 & USA & & \\
\hline MT259253 & USA & MT184911 & USA & & \\
\hline MT259254 & USA & MT184912 & USA & & \\
\hline MT253701 & China & MT184913 & USA & & \\
\hline MT253702 & China & MT184910 & USA & & \\
\hline MT253703 & China & MT184908 & USA & & \\
\hline MT253704 & China & MT184907 & USA & & \\
\hline MT253706 & China & MT159708 & USA & & \\
\hline MT253707 & China & MT159715 & USA & & \\
\hline MT253710 & China & MT159718 & USA & & \\
\hline MT253705 & China & MT159720 & USA & & \\
\hline MT253708 & China & MT159721 & USA & & \\
\hline MT253709 & China & MT159722 & USA & & \\
\hline
\end{tabular}




\section{References}

${ }_{85}$ [1] L. van der Hoek, K. Pyrc, M. F. Jebbink, W. Vermeulen-Oost, R. J. Berkhout, K. C. Wolthers, P. M. Wertheim-van Dillen, J. Kaandorp, J. Spaargaren, B. Berkhout, Identification of a new human coronavirus, Nature medicine 10 (4) (2004) 368-373.

[2] K. V. Holmes, Sars-associated coronavirus, New England Journal of 9o $\quad$ Medicine 348 (20) (2003) 1948-1951.

[3] L. Mousavizadeh, S. Ghasemi, Genotype and phenotype of covid-19: Their roles in pathogenesis, Journal of Microbiology, Immunology and Infection (2020).

[4] P. Yang, X. Wang, Covid-19: a new challenge for human beings, Cellular \& Molecular Immunology (2020) 1-3.

[5] C. Li, Y. Yang, L. Ren, Genetic evolution analysis of 2019 novel coronavirus and coronavirus from other species, Infection, Genetics and Evolution (2020) 104285.

[6] R. Lu, X. Zhao, J. Li, P. Niu, B. Yang, H. Wu, W. Wang, H. Song, B. Huang, N. Zhu, et al., Genomic characterisation and epidemiology of 2019 novel coronavirus: implications for virus origins and receptor binding, The Lancet 395 (10224) (2020) 565-574.

[7] P. D. Yadav, V. A. Potdar, M. L. Choudhary, D. A. Nyayanit, M. Agrawal, S. M. Jadhav, T. D. Majumdar, A. Shete-Aich, A. Basu, P. Abraham, et al., Full-genome sequences of the first two sars-cov-2 viruses from india., The Indian Journal of Medical Research (2020).

[8] M. Lipsitch, D. L. Swerdlow, L. Finelli, Defining the epidemiology of covid19 - studies needed, New England Journal of Medicine (2020). 
[9] A. S. Fauci, H. C. Lane, R. R. Redfield, Covid-19-navigating the uncharted (2020).

[10] W. Liu, Q. Zhang, J. Chen, R. Xiang, H. Song, S. Shu, L. Chen, L. Liang, J. Zhou, L. You, et al., Detection of covid-19 in children in early january 2020 in wuhan, china, New England Journal of Medicine (2020).

[11] F. Jiang, L. Deng, L. Zhang, Y. Cai, C. W. Cheung, Z. Xia, Review of the clinical characteristics of coronavirus disease 2019 (covid-19), Journal of General Internal Medicine (2020) 1-5.

[12] J. Stebbing, A. Phelan, I. Griffin, C. Tucker, O. Oechsle, D. Smith, P. Richardson, Covid-19: combining antiviral and anti-inflammatory treatments, The Lancet Infectious Diseases (2020).

[13] J. F.-W. Chan, C. C.-Y. Yip, K. K.-W. To, T. H.-C. Tang, S. C.-Y. Wong, K.-H. Leung, A. Y.-F. Fung, A. C.-K. Ng, Z. Zou, H.-W. Tsoi, et al., Improved molecular diagnosis of covid-19 by the novel, highly sensitive and specific covid-19-rdrp/hel real-time reverse transcription-polymerase chain reaction assay validated in vitro and with clinical specimens, Journal of Clinical Microbiology (2020).

[14] S. Zhang, M. Y. Diao, L. Duan, Z. Lin, D. Chen, The novel coronavirus (sars-cov-2) infections in china: prevention, control and challenges, Intensive Care Medicine (2020) 1-3.

[15] C. Sohrabi, Z. Alsafi, N. O’Neill, M. Khan, A. Kerwan, A. Al-Jabir, C. Iosi-

130 fidis, R. Agha, World health organization declares global emergency: A review of the 2019 novel coronavirus (covid-19), International Journal of Surgery (2020). 
[16] P. Sun, X. Lu, C. Xu, W. Sun, B. Pan, Understanding of covid-19 based on current evidence, Journal of Medical Virology (2020).

${ }_{135}$ [17] J. K. Das, P. P. Choudhury, A. Chaudhuri, S. S. Hassan, P. Basu, Analysis of purines and pyrimidines distribution over mirnas of human, gorilla, chimpanzee, mouse and rat, Scientific reports 8 (1) (2018) 1-19.

[18] J. P. Banerjee, J. K. Das, P. P. Choudhury, S. Mukherjee, S. S. Hassan, P. Basu, The variations of human mirnas and ising like base pairing models, BioRxiv (2018) 319301.

[19] S. R. Schaecher, J. M. Mackenzie, A. Pekosz, The orf7b protein of severe acute respiratory syndrome coronavirus (sars-cov) is expressed in virusinfected cells and incorporated into sars-cov particles, Journal of virology 81 (2) (2007) 718-731.

${ }_{145}$ [20] A. Wu, Y. Peng, B. Huang, X. Ding, X. Wang, P. Niu, J. Meng, Z. Zhu, Z. Zhang, J. Wang, et al., Genome composition and divergence of the novel coronavirus (2019-ncov) originating in china, Cell host \& microbe (2020). 